SANTOS, A.M.; BARBOSA, A.B.; ROCHA, T.J.M.; PIMENTEL, E.C.; RODRIGUES, M.M.L.

REF-ISSN1808-0804 Vol.XI (3),20-31, 2014.

\title{
AVALIAÇÃO DE TESTE RAPIDO PARA HIV, PADRONIZADO PELO MINISTÉRIO DA SAÚDE, UTILIZADO EM UMA MATERNIDADE ESCOLA EM MACEIÓ-AL
}

ASSESSMENT FOR HIV RAPID TEST STANDARD FOR MINISTRY OF HEALTH, MATERNITY USED IN A SCHOOL IN MACEIO-AL

EVALUACIÓN DE PRUEBA RÁPIDA PARA VIH, ESTÁNDAR POR EL MINISTERIO DE SALUD, SE USA EN UNA ESCUELA EN LA MATERNIDAD MACEIÓ-AL

\section{Adriano Mateus Santos, Allam Bezerra Barbosa, Thiago José Matos Rocha, Emanuelle Cavalcante Pimentel, Mônica Meira Leite Rodrigues}

Recebido em 29/12/2013, Aceito em 25/09/2014.

RESUMO: O teste rápido foi considerado um teste de triagem para infecção pelo HIV durante muito tempo. A presente pesquisa teve o objetivo de avaliar os testes rápidos para detecção de HIV padronizados pelo Ministério da saúde, utilizados em uma maternidade escola em Maceió AL. Foi realizado um estudo epidemiológico descritivo transversal utilizando o banco de dados de gestantes que buscaram atendimento na Maternidade Escola Santa Mônica da cidade de Maceió-Alagoas. Das 764 gestantes submetidas ao teste rápido para diagnóstico da infecção por HIV-1 e HIV-2, 8 pacientes (1,05\%) apresentaram positividade nos testes rápidos utilizados (Bio manguinhos e Rapid check). A faixa etária mais frequente das pacientes soropositivas para HIV foi 20-29 anos de idade. Os resultados da presente pesquisa evidenciam que a triagem de infecção por HIV-1 e HIV-2 pelo teste rápido é uma excelente opção para maternidades que atendem parturientes com considerada taxa da infecção pelo HIV-1 e HIV-2, porém devem ser realizados dois testes para maior confiabilidade.

PALAVRAS-CHAVE: HIV, Gestantes, Teste rápido 


\section{i) Revista Eletrônica de Farmácia}

SANTOS, A.M.; BARBOSA, A.B.; ROCHA, T.J.M.; PIMENTEL, E.C.; RODRIGUES, M.M.L.

REF-ISSN1808-0804 Vol.XI (3),20-31, 2014.

ABSTRACT: The rapid test was considered a screening test for HIV infection for a long time. This study aimed to evaluate the rapid tests for detecting HIV standardized by the Ministry of Health, used in a Maternity Hospital in Maceió-AL. The cross-sectional epidemiological study using the database of women who sought care in the Maternity Hospital Santa Monica city of Maceió-AL. Of the 764 pregnant women undergoing the rapid test for diagnosis of HIV-1 e HIV-2 infection, 8 patients $(1.05 \%)$ were positive in rapid tests used (Bio Manguinhos and Rapid check). The most frequent age range of HIV-positive patients was 20-29 years old. The results of this study show that screening for HIV-1 and HIV-2 for the rapid test is an excellent choice for hospitals that serve pregnant women considered the rate of infection with HIV-1 and HIV-2, but must be made two tests for reliability.

KEYWORDS: HIV, Pregnancy, Quick Test

RESUMÉN: La prueba se consideró una prueba de detección rápida de la infección por VIH a largo. Esta investigación tuvo como objetivo evaluar las pruebas rápidas para la detección del VIH estandarizada por el Ministerio de Salud, que se utilizan en una escuela de maternidad en Maceio AL. Se realizó un estudio epidemiológico descriptivo transversal utilizando la base de datos de las mujeres que buscaron atención en el Santa Monica Escuela de Maceió-Alagoas maternidad. De las 764 mujeres embarazadas se sometieron a la prueba rápida para el diagnóstico del VIH1 y VIH-2, 8 pacientes (1,05\%) fueron positivos en las pruebas rápidas utilizado (Bio Manguinhos Rápido y comprobar). La edad más frecuente de los pacientes seropositivos para VIH fue 20 a 29 años de edad. Los resultados de esta investigación evidencia que la detección de VIH-1 y VIH-2 por la prueba rápida es una excelente opción para los hospitales que atienden a las mujeres embarazadas consideradas con tasa de $\mathrm{VIH}-1$ y $\mathrm{VIH}-2$, pero se debe realizar dos pruebas de fiabilidad.

PALABRAS CLAVES: VIH, las mujeres embarazadas, la prueba rápida 


\section{i) Revista Eletrônica de Farmácia}

SANTOS, A.M.; BARBOSA, A.B.; ROCHA, T.J.M.; PIMENTEL, E.C.; RODRIGUES, M.M.L.

REF-ISSN1808-0804 Vol.XI (3),20-31, 2014.

\section{INTRODUÇÃO}

O crescimento da epidemia de HIV (sigla em inglês do virus da imunodeficiência humana) na população heterossexual brasileira foi acompanhado do aumento considerável da incidência em mulheres. Com isso houve aumento do número de crianças infectadas pelo HIV, por transmissão vertical. Aproximadamente $84 \%$ dos casos de AIDS (sigla em inglês da Síndrome da Imunodeficiência Adquirida) em crianças brasileiras são atribuídos à transmissão vertical ${ }^{1-2}$.

Erros ocorridos na detecção precoce da infecção pelo vírus do HIV durante o pré-natal limitam as possibilidades de redução da incidência de transmissão vertical do vírus. Em sua maioria essas mulheres não tem acesso ao teste do HIV devido a sua condição social ou por falhas no sistema de saúde ${ }^{3-4}$.

Embora no Brasil testes existam para determinar a soropositividade para HIV, as crianças continuam a ser infectadas. Isso ocorre devido ao fato de muitas mulheres chegarem às maternidades sem terem realizado o pré-natal, além da cobertura do teste para a infecção pelo HIV durante o pré-natal está abaixo de $40 \%$ no Brasil. Logo, para a maioria das mulheres soropositivas para HIV a única oportunidade de terem acesso ao diagnóstico e tratamento da transmissão vertical é por ocasião da internação para o parto $^{5-6}$.

O emprego de testes rápidos para o diagnóstico de infecção pelo HIV na gestação, tem se tornado um método diagnóstico rápido e eficaz dessa infecção durante a assistência ao pré-natal e ao parto. O uso destes testes é rotina em maternidades brasileiras, apesar de nem sempre estarem disponíveis. São muito importantes em áreas com baixa adesão ao pré-natal e com poucos recursos diagnósticos ${ }^{4}$.

Os testes rápidos foram desenvolvidos no final da década de 80 e ganharam popularidade no começo dos anos 90. Atualmente, apresentam sensibilidade variando entre 99,59 e $100 \%$ e especificidade entre 99,71 e $100 \%$, semelhantes aos testes de Elisa de terceira geração ${ }^{7}$.

O teste rápido constitui de um ensaio imunocromatográfico utilizado para a detecção qualitativa de anticorpos HIV-1 e HIV-2 em sangue total, soro ou plasma humano, possui 


\section{i) Revista Eletrônica de Farmácia}

SANTOS, A.M.; BARBOSA, A.B.; ROCHA, T.J.M.; PIMENTEL, E.C.; RODRIGUES, M.M.L.

REF-ISSN1808-0804 Vol.XI (3),20-31, 2014.

rapidez e fácil interpretação no resultado ${ }^{5,7}$.

As vantagens dos testes a que se refere, são: ser um produto de fácil manuseio; não exigir infraestrutura laboratorial; poder ser realizado por profissional de saúde de nível assistencial, desde que o mesmo esteja capacitado; e o resultado ser obtido em menos de 30 minutos, otimizando, desta forma, a ida do usuário ao serviço de saúde ${ }^{8}$.

Um estudo realizado por Kassler et al. ${ }^{9}$ nos centros de controle e prevenção de DSTs nos Estados Unidos, mostrou que não houve diferença significativa entre os resultados de teste rápido e teste Western blot para o diagnóstico do HIV.

Segundo Carvalho et al. $^{3}$ a realização do teste rápido para HIV na admissão à maternidade é algo muito importante, devendo destacar que sua realização deve ser proposta para as gestantes não testadas previamente, sendo fundamental a realização voluntária e confidencial do teste.

O teste rápido foi considerado um teste de triagem durante muito tempo. A Portaria No 34 da Secretaria de Vigilância em Saúde (SVS) e Ministério da Saúde (MS) publicada em Julho de 2005 e a Portaria No 151/SVS/MS publicada em outubro de 2009 regulamenta o uso dos testes rápidos para o diagnóstico da infecção pelo HIV ${ }^{10-11}$. A implantação dessa estratégia em locais de difícil acesso aos serviços de saúde vem agregar um inestimável valor à qualidade do serviço oferecido, promovendo a melhoria da assistência nesses serviços de saúde. Ainda de acordo com a Portaria No 151/SVS/MS todas as parturientes devem realizar o teste rápido.

Devido à ausência de dados na literatura sob soropositividade para HIV em gestantes no estado de Alagoas, bem como de estudos comparando os testes rápidos para detecção de HIV padronizados pelo Ministério da Saúde, faz-se necessária à realização da pesquisa acerca deste tema. Uma vez que a maioria da população feminina têm seus partos realizados na rede pública hospitalar, o momento do parto assume importância estratégica e privilegiada como momento de recuperação das oportunidades perdidas durante o prénatal no que se refere ao controle da Transmissão Vertical do HIV e da sífilis congênita, o estudo foi realizado 
SANTOS, A.M.; BARBOSA, A.B.; ROCHA, T.J.M.; PIMENTEL, E.C.; RODRIGUES, M.M.L.

REF-ISSN1808-0804 Vol.XI (3),20-31, 2014.

em uma maternidade publica de Maceió-AL.

\section{MATERIAL E MÉTODO}

Foi realizado um estudo do tipo epidemiológico, descritivo e transversal com 764 resultados de testes rápidos para HIV-1 e HIV-2 realizados por todas as parturientes atendidas na Maternidade Escola Santa MônicaMaceió/AL no período de 01 de janeiro a 30 de abril de 2011. As variáveis estudadas foram: faixa etária, resultados dos testes rápidos biomanguinhos e testes confirmatórios (Rapid Check e Western Blot). Para todos os casos inconclusivos foi realizado teste de Western Blot. A estatística utilizada foi do tipo descritivo (usando-se percentual) e inferencial (com o uso do teste de concordância kappa), as quais foram feitas no programa Microsoft Office Excel $2007^{\circledR}$. Foram preservados em anonimato os nomes de mães soropositivas para o vírus HIV e crianças expostas, bem como de todas as puérperas que foram entrevistadas. O trabalho foi aprovado pelo comitê de ética em pesquisa da
Universidade Estadual de Ciências da Saúde de Alagoas sob protocolo de $n^{\circ}$ 1693, o Termo de Consentimento Livre e Esclarecido não se aplica, pois o trabalho foi desenvolvido através de análise de banco de dados da própria maternidade.

\section{RESULTADOS}

Na Maternidade Escola Santa Mônica, como é preconizada pela Portaria No 151/SVS/MS a realização de teste rápido faz parte da triagem das parturientes ${ }^{11}$. No período estudado, todas as 764 gestantes atendidas foram submetidas ao teste rápido para diagnóstico da infecção por HIV-1/2 antes da realização do parto. Os testes utilizados foram das marcas Bio-manguinhos e Rapid check (Quadro 1). 


\section{- Revista Eletrônica}

SANTOS, A.M.; BARBOSA, A.B.; ROCHA, T.J.M.; PIMENTEL, E.C.; RODRIGUES, M.M.L.

REF-ISSN1808-0804 Vol.XI (3),20-31, 2014.

\begin{tabular}{|c|c|c|c|c|c|}
\hline Testes & $\begin{array}{c}\text { Anticorpos } \\
\text { pesquisados }\end{array}$ & Metodologia & Amostra & Sensibilidade & Especificidade \\
\hline $\begin{array}{c}\text { Biomangui } \\
\text { nhos }\end{array}$ & $\begin{array}{c}\text { anti- } \\
\text { gp41/120/ } \\
36\end{array}$ & $\begin{array}{l}\text { Imunocromatográfi } \\
\text { co }\end{array}$ & $\begin{array}{c}\text { Soro, } \\
\text { plasma } \\
\text { e } \\
\text { sangue } \\
\text { total. }\end{array}$ & $99,77 \%$ & $100 \%$ \\
\hline $\begin{array}{l}\text { Rapid } \\
\text { check }\end{array}$ & $\begin{array}{c}\text { anti- } \\
\text { gp41/120/ } \\
36\end{array}$ & $\begin{array}{l}\text { Imunocromatográfi } \\
\text { co }\end{array}$ & $\begin{array}{c}\text { Soro, } \\
\text { plasma } \\
\text { e } \\
\text { sangue } \\
\text { total. }\end{array}$ & $100 \%$ & $98,94 \%$ \\
\hline
\end{tabular}

Quadro 1 - Caracterização dos testes para pesquisa da infecção pelo vírus HIV-1 e HIV-2 na Maternidade Escola Santa Mônica no período de 01 de janeiro a 30 de abril de 2011.

Dos 764 resultados analisados, 10 $(1,31 \%)$ apresentaram positividade pelo teste rápido Bio-manguinhos, sendo que $2(0,26 \%)$ destes, após realização do segundo teste confirmatório, Rapid Check, apresentaram resultados negativos, sendo portanto considerados inconclusivos (Tabela 1). O teste Rapid Check confirmou, portanto, 8 $(1,05 \%)$ testes positivos, e os dois que deram inconclusivos anteriormente, foram confirmados com Western Blot. Os testes tiveram grande concordância, com valor de kappa 0,99.

\begin{tabular}{|c|c|c|c|c|}
\hline \multirow{2}{*}{$\begin{array}{l}\text { Teste } \\
\text { Resultado }\end{array}$} & \multicolumn{2}{|c|}{ Teste rápido Bio-Manguinhos } & \multicolumn{2}{|c|}{ Rapid check } \\
\hline & $\mathbf{N}$ & $\%$ & $\mathbf{N}$ & $\%$ \\
\hline Soropositividade & 10 & 1,31 & 8 & 1,05 \\
\hline Soronegatividade & 754 & 98,69 & 756 & 98,95 \\
\hline Total & 764 & 100 & 764 & 100 \\
\hline
\end{tabular}

Fonte: Dados da pesquisa.

Tabela 1 - Resultados dos testes rápidos para detecção do HIV realizados na Maternidade Escola Santa Mônica no período de 01 de janeiro a 30 de abril de 2011. 


\section{O) Revista Eletrônica}

SANTOS, A.M.; BARBOSA, A.B.; ROCHA, T.J.M.; PIMENTEL, E.C.; RODRIGUES, M.M.L.

REF-ISSN1808-0804 Vol.XI (3),20-31, 2014.

A faixa etária mais frequente das pacientes soropositivas para HIV deste estudo, foi a de 20-29 anos de idade (71,5\%) como mostra o gráfico 1 .

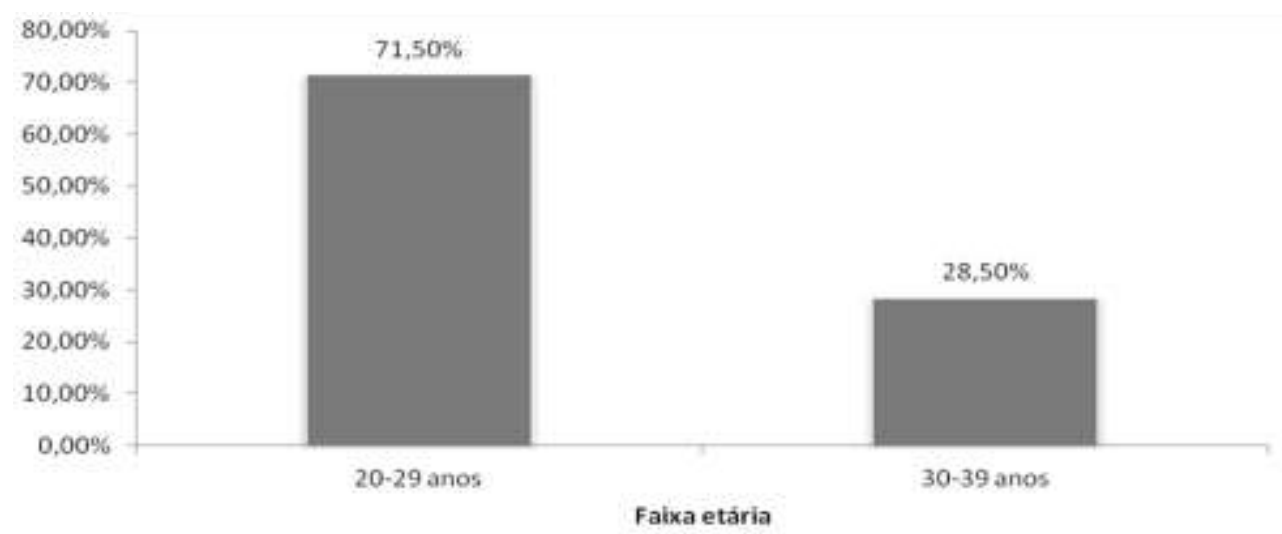

Gráfico 1 - Faixa etária das pacientes com teste rápido para HIV-1 positivo atendidas na Maternidade Escola Santa Mônica.

Fonte: Dados da pesquisa.

Este dado está de acordo com estudos realizados 5,12-13. Porém, não corrobora com os resultados de pesquisa desenvolvida em Maceió-AL, onde a faixa etária com maior frequência de soropositividade foi de 30-39 anos ${ }^{14}$.

DISCUSSÃO

A transmissão vertical é a principal via de infecção pelo HIV na população infantil ${ }^{3,15}$. No Brasil, esta forma de transmissão é responsável por mais de $90 \%$ dos casos notificados de $\operatorname{AIDS}^{2,7}$.

É extramamente importante a realização do diagnóstico da infecção pelo HIV e sífilis em parturientes, mesmo que tardiamente, durante o trabalho de parto. O início da terapia antiretroviral quanto mais cedo iniciado diminui as chances de contaminação do feto ${ }^{16-17}$.

Assim, estudos planejados estão sendo conduzidos para avaliar diferentes maneiras de reduzir a transmissão do HIV da mãe para o filho. Nesse sentido, o Ministério da Saúde orienta a atenção integral à saúde na perspectiva das Redes de Atenção à Saúde (RAS). Na perspectiva de promoção e garantia do direito à saúde das mulheres em todos os ciclos de vida, diferentes faixas etárias e das distintas 


\section{i) Revista Eletrônica de Farmácia}

SANTOS, A.M.; BARBOSA, A.B.; ROCHA, T.J.M.; PIMENTEL, E.C.; RODRIGUES, M.M.L.

REF-ISSN1808-0804 Vol.XI (3),20-31, 2014.

populações, raça, etnia, e pela ampliação do enfoque da saúde sexual e da saúde reprodutiva, lançou em 2011 a Rede Cegonha ${ }^{18}$.

A rede Cegonha visa assegurar à mulher o direito ao planejamento reprodutivo e à atenção humanizada à gravidez, ao parto e ao puerpério, bem como à criança o direito ao nascimento seguro e ao crescimento e ao desenvolvimento saudáveis ${ }^{18}$.

Como rotina já estabelecida pelo Ministério da Saúde, na primeira consulta de pré-natal o profissional de saúde deve solicitar sorologia antiHIV, com consentimento da mulher após o aconselhamento pré-teste. Em Maceió-AL, as maternidades do Hospital Universitário Alberto Antunes (HU), Maternidade Escola Santa Mônica e na Casa Maternal Denilma Bulhões realizam este fluxo após consentimento da mulher.

A descoberta de métodos diagnósticos precisos, acessíveis do ponto de vista de custeio e tecnologia é necessária para a redução $\mathrm{da}$ transmissão vertical do HIV. Apesar dos avanços conseguidos, ELISA não permite resultados rápidos, limitando seu uso na identificação de pacientes em situação de urgência diagnóstica, como a gestante em trabalho de parto. Para esta finalidade foram desenvolvidos os "testes rápidos", que são mais simples, rápidos e de baixo custo. Sendo assim, permitem a identificação das gestantes portadoras do vírus e institui medidas para reduzir a transmissão vertical desse vírus, pois apresentam alta especificidade e sensibilidade ${ }^{19}$.

Um estudo realizado por Carvalho et al. $^{3}$ com gestantes internadas em trabalho de parto em 2002, os resultados demonstraram que entre as 298 gestantes avaliadas, o teste rápido foi positivo em 16 pacientes $(5,3 \%)$, mostrando que estes dados revelam o valor do teste rápido para a detecção de infecção por HIV em situações de emergência, como o parto, de gestantes não testadas previamente.

Sensibilidade é definida como sendo a proporção de indivíduos com a doença que apresentam o teste positivo, já especificidade é a proporção de indivíduos sem a doença que apresentam o teste negativo. A sensibilidade e a especificidade de um teste são propriedades que servem para tomada de decisão sobre a utilização ou não de um teste para diagnóstico. Seus valores dão a probabilidade de que o teste seja 


\section{i) Revista Eletrônica de Farmácia}

SANTOS, A.M.; BARBOSA, A.B.; ROCHA, T.J.M.; PIMENTEL, E.C.; RODRIGUES, M.M.L.

REF-ISSN1808-0804 Vol.XI (3),20-31, 2014.

positivo ou negativo, em pessoas sabidamente doentes ou não doentes respectivamente ${ }^{20}$.

Estudos

anteriormente

realizados mostram percentuais de positividade abaixo dos obtidos na presente pesquisa. A prevalência de parturientes com teste rápido reagente em três maternidades de Sergipe $^{5}$ foi de $0,42 \%, 0,7 \%$ na maternidade referência do estado do Pará ${ }^{17}$ e de $0,88 \%$ com parturientes de um hospital público de Maceió, Alagoas ${ }^{14}$.

Estudo realizado por Castro et al. ${ }^{12}$, no Serviço de Obstetrícia do Hospital Geral de Caxias do Sul, mostra positividade em $0,53 \%$ do total das gestantes atendidas no referido Serviço no período de março de 1998 a novembro de 2000.

De acordo com o Ministério da Saúde $^{5}$, anualmente 3 milhões de mulheres dão à luz no Brasil, sendo que a prevalência de infecção por HIV é de $0,42 \%$ das mulheres no momento do parto, aproximadamente 13 mil grávidas ${ }^{11}$.

Estudos revelam que a detecção do vírus HIV-1 em gestantes por meio de testes rápidos possibilita o tratamento profilático da transmissão vertical com o uso de antirretrovirais, reduzindo assim os custos com crianças infectadas, principalmente em pacientes que não realizaram o pré-natal ${ }^{21-23}$.

A triagem da infecção por HIV1 e HIV-2 pelos testes rápidos normatizados pela portaria 151 é uma excelente opção para maternidades que atendem parturientes com considerada taxa da infecção pelo HIV, principalmente porque muitas destas não realizaram teste sorológico no pré-natal.

Apesar dos testes padronizados pelo Ministério da Saúde apresentar alta sensibilidade e especificidade para detecção do HIV-1 e HIV-2, a segurança do diagnóstico aumenta quando são realizados os dois testes rápidos em paralelo, sendo necessária a utilização de um terceiro teste para confirmar os testes positivos.

Torna-se necessário oferecer o teste rápido para o HIV e sífilis nas maternidades com a realização de Aconselhamento pré e pós-teste, devendo-se ressaltar que sua realização deve ser proposta para as gestantes não testadas previamente, sendo fundamental realização voluntária e confidencial. É de suma importância, para a efetiva implementação desta prática, a 


\section{- Revista Eletrônica de Farmácia}

SANTOS, A.M.; BARBOSA, A.B.; ROCHA, T.J.M.; PIMENTEL, E.C.; RODRIGUES, M.M.L.

REF-ISSN1808-0804 Vol.XI (3),20-31, 2014.

necessidade de qualificação dos profissionais de saúde, através da capacitação e da disponibilidade de insumos necessários.

\section{REFERÊNCIAS}

Sbalqueiro RL, Reggiani C, Tristão EG, Urbanetz AA, Andrande RP, Nascimento DJ. Estudo da prevalência e variáveis epidemiológicas da infecção pelo HIV em gestantes atendidas na maternidade do hospital de Clínicas de Curitiba. J Bras Doenças Sex Transm, 2004; 16(2): 40-47.

Santos EM, Reis AC, Westman S, Alves RG. Avaliação do grau de implantação do programa de controle da transmissão vertical do HIV em maternidades do "Projeto Nascer". Epidemiol Serv Saúde, 2010; 19(3): 257-269.

Carvalho RL, Krahe C, Farina G, Paula DO, Richetti N, Crossetti T. Teste rápido para diagnóstico da infecção pelo HIV em parturientes. Rev Bras Ginec Obst, 2004; 26(4): 325-328.

Miranda AE, Rosetti Filho E, Trindade CR, Gouvêa GM, Costa DM, Oliveira T G, França LC, Dietze R. Prevalência de sífilis e HIV utilizando testes rápidos em parturientes atendidas nas maternidades públicas de Vitória, Estado do Espírito Santo. Rev Soc Bras Med Trop, 2009; 42(4): 386-391.

Lemos LMD, Gurgel RQ, Fabbro ALD. Prevalência da infecção por HIV em parturientes de maternidades vinculadas ao SUS. Rev Bras Ginecol Obstet, 2005; 27(1): 32-36.

Veloso VG, Bastos FI, Portela MC, Grinsztejn B, João EC, Pilotto JHS et al. HIV rapid testing as a key strategy for prevention of mother-to-child transmission in Brazil. Rev Saúde Pública, 2010; 44(5): 803-811.

Marques WP, Sáez-Alquézar A, Ferreira HG, Requejo ACSP, Figueiredo CG S. Avalianção de um teste rápido para detecção de anticorpos anti-HIV. NewsLab, 2007; 84:176-184.

Okamura M, Santos EM, Cruz MM, Vasconcelos AL. Avaliação da implantação do teste rápido para diagnóstico do HIV no Estado do Amazonas. Rev Eletrônica Portas, 2008; 2(2): 41-62.

Kassler WJ, Haley C, Jones WK, Gerber AR, Kennedy EJ, George JR. Performance of a rapid, on-site human immunodeficiency virus antibody assay in a public health setting. J Clin Microbiol, 1995; 33:2899-2902.

Brasil. Ministério da Saúde. Regulamentação do uso de testes rápidos para diagnóstico da infecção pelo HIV em situações especiais. Portaria no 34 de Julho de 2005, publicada no Diário Oficial da União. Brasília (DF); 2005. [acesso 23 maio 2014] Disponível em: http://portal.saude.gov.br/portal/arquivos/pdf/portaria34.pdf 


\section{- Revista Eletrônica de Farmácia}

SANTOS, A.M.; BARBOSA, A.B.; ROCHA, T.J.M.; PIMENTEL, E.C.; RODRIGUES, M.M.L.

REF-ISSN1808-0804 Vol.XI (3),20-31, 2014.

Brasil. Ministério da Saúde. Regulamentação do uso de testes rápidos para diagnóstico da infecção pelo HIV em situações especiais. Portaria no 151 de Outubro de 2009, publicada no Diário Oficial da União. Brasília (DF); 2009. [acesso 23 maio 2014] Disponível em: http://www.brasilsus.com.br/legislacoes/svs/100904-151.html

Castro TPT, Lorenzi DRS, Tonin C, Zapparolli M. HIV e gestação. Rev Cient, 2001; 10(1): 39-46.

Sanz SM, Guinsburg R. Prevalência da soropositividade HIV e sífilis parágrafo los gestantes de hum hospital de Referência Materno Infantil do Estado do Pará. Rev Para Med, 2008; 22 (3):1-11.

Costa CRS, Maia ES, Rodrigues MML. Frequência de testes rápidos positivos para HIV em um Hospital Público de Alagoas. [Trabalho de Conclusão de Curso]. Maceió $(A L)$ : Centro Universitário Cesmac; 2010.

Castilho EA, Bastos FI, Scwarcwald CL, Fonseca MGM. A AIDS no Brasil: uma epidemia em mutação. Cad Saúde Publica, 2000; 16 (Suppl 1):4-5.

Rodrigues CS, Guimarães MDG; Grupo Nacional de Estudo sobre Sífilis Congênita. Positividade para sífilis em puérperas: ainda um desafio para o Brasil. Rev Pan Saúde Pública, 2004; 16:168-175.

Reiche EM, Morimoto HK, Farias GN, Hisatsugu KR, Geller L, Gomes AC, Inoue HY, Rodrigues G, Matsuo T. Prevalência de tripanossomíase americana, sífilis, toxoplasmose, rubéola, hepatite $B$, hepatite $C$ e da infecção pelo vírus da imunodeficiência humana, avaliada por intermédio de testes sorológicos, em gestantes atendidas no período de 1996 a 1998 no Hospital Universitário Regional Norte do Paraná. Rev Soc Bras Med Trop, 2000, 33:519-527.

Brasil. Ministério da saúde. Gabinete do Ministro. Portaria no 1.459, de 24 de junho de 2011. [acesso 23 agosto 2014] Disponível em:

<http://bvsms.saude.gov.br/bvs/saudelegis/gm/2011/prt1459_24_06_2011.html>

Duarte G, Gonçalves CV, Marcolin AC, Paschoini MC, Quintana SM, Mussi-Pinhata MM. Teste rápido para detecção da infecção pelo HIV-1 em gestantes. Rev Bras Ginecol Obstet, 2001; 23(02):107-111.

Fletcher RH, Fletcher SW, Wagner EH. Epidemiologia clínica: elementos essenciais. 3a edição. Porto Alegre, 1996.

Grobman WA, Garcia PM. The cost-effectiveness of voluntary intrapartum rapid human immunodeficiency virus testing for women without adequate prenatal care. Am J Obstet Gynecol 1999; 181:1062-71.

Gaddis GM, Gaddis ML. Introduction to biostatistics: Part 3, Sensitivity, Specifcity, Predictive Value, and Hypothesis Testing. Annals of Emergency Medicine. 1990; 19:591-596. 
SANTOS, A.M.; BARBOSA, A.B.; ROCHA, T.J.M.; PIMENTEL, E.C.; RODRIGUES, M.M.L.

REF-ISSN1808-0804 Vol.XI (3),20-31, 2014.

Stringer JS, Rouse DJ. Rapid testing and zidovudine treatment to prevent vertical transmission of human immunodeficiency virus in unregistered parturients: a costeffectiveness analysis. Obstet Gynecol, 1999; 94:34-40. 